\title{
L'armée de l'an II : la levée en masse et la création d'un mythe républicain
}

\section{Alan Forrest}

\section{(2) OpenEdition}

1 Journals

\section{Édition électronique}

URL : https://journals.openedition.org/ahrf/1385

DOI : 10.4000/ahrf.1385

ISSN : 1952-403X

Éditeur :

Armand Colin, Société des études robespierristes

\section{Édition imprimée}

Date de publication : 1 mars 2004

Pagination : 111-130

ISSN : 0003-4436

\section{Référence électronique}

Alan Forrest, «L'armée de l'an II : la levée en masse et la création d'un mythe républicain », Annales historiques de la Révolution française [En ligne], 335 I janvier-mars 2004, mis en ligne le 20 février 2008 consulté le 22 avril 2022. URL : http://journals.openedition.org/ahrf/1385 ; DOI : https://doi.org/ 10.4000/ahrf.1385

Ce document a été généré automatiquement le 22 avril 2022.

Tous droits réservés 


\title{
L'armée de l'an II : la levée en masse et la création d'un mythe républicain
}

\author{
Alan Forrest
}

1 Parmi les nombreux aspects de la Révolution française destinés à devenir des points de référence pour les générations futures, l'armée occupe une place particulière. Elle devient un thème de commémoration récurrent aux XIX ${ }^{\mathrm{e}}$ et $\mathrm{XX}^{\mathrm{e}}$ siècles ${ }^{1}$. Les raisons, nombreuses, ne sont pas toutes liées à l'idéologie de la Révolution ni, les années passant, à l'approbation de son œuvre. Car l'image d'une armée héroïque et victorieuse attire des obédiences différentes dans la France post-révolutionnaire et post-impériale et elle se décline dans un grand nombre de registres opposés. Pour certains, elle représente bien sûr un paradigme des valeurs révolutionnaires, une armée fondée sur les principes d'égalité et de citoyenneté, dont les soldats manifestent leur zèle et leur engagement pour la cause au nom de laquelle ils se battent - mais cette représentation ne va pas sans poser de problème, comme nous le verrons. Pour d'autres, elle incarne le nationalisme à une époque où le renouveau et les élans du romantisme encouragent à une renaissance du sentiment national et au développement du débat sur l'identité française. Le courage et l'élan des troupes révolutionnaires symbolisent les valeurs nationales. Ces caractères constitutifs de l'esprit guerrier français permettent de distinguer la France de l'Allemagne et d'autres États modernes ; ils contribuent, au XIX ${ }^{e}$ siècle, à la définition de la francité. En focalisant par dessus tout leur discours sur la détermination et les exploits héroïques de l'armée, les nationalistes détournent l'attention des citoyens pour la politique. Comme symbole, et même comme trope, l'armée de l'an II est vue pour ce qu'elle n'est pas et a été utilisée par des Français d'appartenances politiques diverses afin d'unifier le peuple de France dans des temps de graves conflits et divisions internes. Républicains radicaux et socialistes revendiquent cette institution comme une part de leur héritage politique. Mais elle attire au-delà de la gauche. Bonapartistes, républicains conservateurs et nationalistes puisent tous dans l'héritage de l'armée révolutionnaire à laquelle ils peuvent aisément s'identifier. Même ceux qui s'alignent aux côtés de l'extrême droite, les plus connus 
relevant de la Ligue des patriotes de Paul Déroulède, s'enorgueillissent de se draper dans le manteau du patriotisme révolutionnaire et se proclament les héritiers des soldats héroïques de l'an II ${ }^{2}$.

Cette même référence aux soldats de l'an II évoque bien sûr un moment très précis dans l'évolution des armées révolutionnaires, durant lequel les troupes incarnent un ensemble particulier de qualités. C'est l'époque où les armées françaises sont formées de jeunes recrues issues de la levée des 300000 hommes et de la levée en masse du printemps et de l'été 1793. Celles-ci ont été ensuite associées par l'amalgame à la vieille armée de ligne afin de créer des demi-brigades victorieuses : elles repoussent l'ennemi au-delà des frontières et entament une guerre de conquête à travers le continent européen. La propagande révolutionnaire nous rapporte que ces hommes sont remarquables pour leur ardeur patriotique, toujours prêts à voler jusqu'aux frontières, à verser la dernière goutte de leur sang par amour et pour la défense de leur pays. En d'autres termes, ils sont de nature et d'esprit différents des autres armées européennes contemporaines, celles composées d'esclaves de rois et de tyrans auxquels elles devaient une obéissance aveugle. Ils sont différents parce qu'ils sont des citoyens, même si en tant que soldats ils ont accepté de renoncer à certains de leurs droits par leur contrat d'engagement. Ce fut un sujet de débat courant dans la France du XVIII ${ }^{\mathrm{e}}$ siècle, notamment sous le règne de Louis XVI, plus précisément dans l'œuvre de Joseph Servan sur Le Soldat citoyen (1780). Ce livre représente le fruit d'une longue campagne menée dans les années 1760 et 1770 contre les abus viscéraux de l'armée d'Ancien Régime. Il critique le fait de dépendre au XVIII ${ }^{\mathrm{e}}$ siècle d' " hommes soudoyés et toujours sur pied ", système peu rentable qui requiert des impôts élevés et s'avère dégradant pour les hommes de ligne. Il en résulte des différences importantes dans les rangs, ce qui nuit à l'efficacité de l'armée. Servan observe qu'il est entouré de manière inefficace, d'une part, "d'officiers supérieurs, quelquefois bornés et minutieux, souvent parvenus par leur naissance plutôt que par leur mérite ", d'autre part, " de bas officiers choisis trop légèrement et trop souvent chargés seuls de la discipline des compagnies », " de soldats indignes souvent d'être citoyens et de les défendre, trop peu payés, mal disciplinés, mal vêtus, mal armés, mal exercés et jamais récompensés " ${ }^{3}$. Il déclare que cela ne peut être corrigé que si le rôle du soldat est avant tout celui d'un citoyen dont l'identité de combattant est reléguée au second plan. Il est convaincu que pour avoir une armée efficace, le soldat doit préserver sa dignité et que cela ne peut s'accomplir que s'il jouit de ses droits de citoyenneté et du respect que lui confèrent ces droits. La société civile et militaire ne fait qu'un, selon Servan. De même, il suggère qu'en dehors du temps des campagnes, les soldats de troupe retournent à la vie civile pour se faire paysans et artisans dans leurs villages jusqu'à ce qu'ils soient rappelés dans leurs unités à l'arrivée du printemps ${ }^{4}$. Ces convictions, propres aux réformateurs et aux critiques de l'Ancien Régime d'avant 1789, obtiennent un large soutien pendant la Révolution française et offrent bien des supports idéologiques aux mutations. Cela permet aux révolutionnaires de définir leurs troupes comme étant composées de volontaires, longtemps après que la réserve effective de volontaires et de gardes nationaux se soit épuisée. On remarque en effet qu'en 1793 il n'y a quasiment plus d'inscriptions volontaires. Beaucoup de soldats qui se sont battus à Valmy et à Jemmapes sont retournés soulagés chez eux à la fin des campagnes, avec le sentiment non dissimulé du devoir accompli et de la nécessité de retourner désormais à leur vie de paysan ou d'artisan ${ }^{5}$. 
3 Le mythe de l'armée de l'an II prend ses racines dans les discours, tant les textes des lois et décrets que les proclamations des politiques et des généraux. Il est entendu dès 1789 que le soldat doit être un citoyen et que l'armée, appelée à défendre la nation, doit lui appartenir et représenter le corps politique qu'elle sert. Les cahiers de doléances ont réclamé des changements quant à la pratique de recrutement, la levée de la milice et au traitement inhumain infligé aux troupes. Des soldats, grisés par les notions de liberté et d'égalité qui circulent déjà dans les casernes, se joignent au débat en envoyant des pétitions à l'Assemblée nationale afin d'obtenir plus de droits, une solde plus élevée et la suppression des punitions dégradantes et cruelles ${ }^{6}$. Mais quels changements envisagent-ils? Comment la notion d'une armée citoyenne est-elle compatible avec la question des libertés individuelles? Déjà, en 1789, le débat à l'Assemblée nationale révèle des points de vue antagonistes sur le sujet ${ }^{7}$. Certains soutiennent que tout service militaire doit être volontaire puisque seuls les volontaires, les hommes acquis à la cause qu'ils défendent et voués au service des armes, peuvent former une force de combat digne du peuple français - c'est par exemple le point de vue de La Rochefoucauld-Liancourt, l'orateur le plus influent sur les questions militaires dans les premiers mois de la Révolution et le défenseur des droits du soldat. Leurs opposants font remarquer qu'il y a cependant une contradiction, car ceux qui se portent volontaires sont en général des hommes sans ressources. Un peuple libre ne peut pas faire confiance pour sa défense à une armée de vagabonds et d'indigents; cela rappellerait les pires abus du XVIII ${ }^{e}$ siècle, lorsque les sergents recruteurs allaient quotidiennement traîner dans les prisons et les dépôts de mendicité pour y chercher des hommes ou, à l'aide de l'alcool, soudoyaient des pauvres hères impressionnables dans les fêtes de village ${ }^{8}$. Les défenseurs de la conscription, comme Dubois-Crancé, ne cessent d'argumenter sur ce point, insistant sur le fait que le peuple est maintenant souverain et que lui seul peut défendre ses libertés si durement acquises. Pour l'heure, il n'est pas question d'envisager un recrutement par la contrainte. Les politiques préfèrent consulter les généraux sur la taille et la composition des armées et faire confiance au flot de volontaires patriotes pour compléter les effectifs de l'armée de ligne héritée de l'Ancien Régime. Mais il s'agit là d'une solution temporaire. À partir de 1792, lorsque le combat devient chose sérieuse, le flot de volontaires s'est tari.

4 En fait, l'armée de l'an II naît à la fois de la nécessité et du désespoir aussi bien que de l'idéalisme, et elle doit moins au volontariat et à la générosité d'esprit qu'à la contrainte. Les hommes, en effet, se sont portés volontaires lors du premier appel, en 1791, mais lors du second, l'année suivante, leur nombre devient nettement insuffisant pour satisfaire les besoins de l'armée. Une certaine forme d'obligation est alors rendue nécessaire et la Révolution, petit à petit, s'oriente vers ce qui conduira à la conscription généralisée de 1799. Ni la levée des 300000 hommes, ni la levée en masse ne peuvent être considérées comme telle : les hommes de chaque classe doivent se présenter d'euxmêmes successivement devant l'officier recruteur, jusqu'à ce que le service militaire devienne un rite de passage pour la jeunesse du pays. Mais ce sont des systèmes fondés sur la contrainte qui comblent les besoins en effectifs de l'armée en imposant des quotas aux départements et, à travers eux, aux districts et aux communes. Certaines circonscriptions - proches des frontières ou ayant une tradition militaire bien établie -, les grandes villes et les plaines agricoles du nord à l'est du pays, trouvent sans grande difficulté les hommes réclamés. Mais beaucoup de jeunes des zones montagneuses refusent de se soumettre à la visite médicale ou vont se cacher dans les bois. Si certains font preuve d'un réel enthousiasme patriotique, d'autres se montrent réfractaires, 
choisissant l'insoumission ou bientôt la désertion - un comportement qui demeure constant sous la Révolution et l'Empire. Aussi, les gendarmes contraignent par la force les jeunes gens au tirage au sort et les parents, frappés d'indignité, sont contraints, s'ils ne livrent pas leurs enfants, de supporter les frais de logement des garnisons. Les maires sont sous la coupe des communautés qu'ils administrent: elles perçoivent les conséquences de la conscription comme un nouvel impôt de sang, levé en l'occurrence sur le sang de leurs enfants. Cela n'aide pas les progrès de la cause révolutionnaire dans les campagnes et est l'une des raisons principales du froid mépris de la loi manifesté par de si nombreuses communautés paysannes. Alors que le mythe insiste sur l'engagement patriotique de la population au nom de la nation et de la Révolution, les maires et les commissaires du gouvernement affrontent au quotidien une réalité bien différente ${ }^{9}$.

Le langage utilisé par les hommes politiques de la Révolution pour débattre de l'effort militaire et parler des hommes de troupe sert à ancrer le mythe tant dans la société civile que dans l'armée : les hommes qui ont donné leur vie et sacrifié leur avenir au service de la République ne peuvent qu'être des hommes de valeur, une image s'opposant à celle, peu reluisante, du soldat ordinaire de l'Ancien Régime. Les politiques se font donc un devoir d'insuffler aux troupes l'héroïsme et le dévouement patriotique, valeurs répétées en des messages aux supports multiformes qui parlent aux armées uniformes et symboles, insignes et musiques militaires, quelque peu distincts des motivations contenues dans les discours patriotiques et les éloges prononcés de manière régulière par leurs chefs ou les représentants en mission à la veille des batailles. La distribution gratuite de journaux radicaux, la présentation de thèmes révolutionnaires dans des pièces de théâtre, la formation de clubs jacobins dans les bataillons et la parution de recueils de chansons révolutionnaires avaient pour but de rappeler aux hommes leurs idéaux, leur rôle de missionnaires politiques se battant pour sauver la France, défendre leurs concitoyens et diffuser un message de liberté dans l'Europe entière ${ }^{10}$. La propagande républicaine insiste sur le fait qu'ils sont des hommes libres, des citoyens qui ont été élus pour accomplir ce grand sacrifice, délaissant certains de leurs droits civiques pour garantir leur liberté et celle de leurs frères: ils s'élèvent ainsi au rang de héros incontestables. Pour Robespierre en particulier, qui soupçonne toujours les officiers et craint les complots au sein des troupes, ces vertus essentielles du soldat ordinaire garantissent la loyauté de l'armée et sont autant de butoirs mis à l'émergence d'un nouveau César ou d'un nouveau Cromwell au sein de l'état-major. Ce soldat est bon comme le peuple est bon, héroïque comme le peuple l'a été en s'élevant contre l'oppression, "bon, grand et généreux » bien que crédule et naïf ${ }^{11}$. Il protège la France de ses ennemis tant extérieurs qu'intérieurs en évitant à la population civile de subir les dérives naturelles d'une armée - un thème qui a depuis été repris par les différentes Républiques soucieuses du maintien de la conscription. Robespierre exhorte la Convention d'éviter avec le plus grand soin tout ce qui peut faire du citoyen-soldat un militaire de carrière, ruinant son civisme dans l'association de la gloire et de l'intérêt personnel ${ }^{12}$.

Cette image entrera dans la légende républicaine et résumera le caractère exclusivement vertueux du soldat de la Première République; elle sera reprise et amplifiée au XIX ${ }^{\mathrm{e}}$ siècle par une nouvelle génération de républicains. Pour beaucoup de ceux-ci l'armée représente la République, une force étroitement associée à la Révolution, à la vertu, à l'identité nationale de la France. L'enthousiasme et la conviction sont au cœur de l'efficacité de l'armée et de ses prouesses militaires. Quand, 
en 1870, la France se trouve à la merci des Prussiens, il est symptomatique que, dans ces heures où nécessité fait loi, la patrie à nouveau en danger s'inspire de la Révolution. Une fois encore, c'est le caractère révolutionnaire de l'infanterie française, son intrépidité, sa spontanéité face à l'injustice, qui sont mis en avant. Le soldat est aussi un révolutionnaire, un militant, qui s'insurge au nom du peuple: Léon Gambetta, en réponse à la crise, n'hésite pas à faire le parallèle entre celui de la Troisième République et celui de la Première, celui de l'an II et le franc-tireur vers lequel la France se tourne à présent. "Citoyens [s'exclamera-t-il] c'est aujourd'hui le 21 septembre. Il y a soixante dix-huit ans à pareil jour nos pères fondaient la République et se juraient à eux-mêmes, en face de l'étranger qui souillait le sol sacré de la patrie, de vivre libres ou de mourir en combattant. Ils ont tenu leur serment; ils ont vaincu, et la République de 1792 est restée dans la mémoire des hommes comme symbole de la grandeur nationale ${ }^{13}$. Mais alors que Gambetta aura raison d'insister sur la nature révolutionnaire de l'armée de l'an II et que son discours sera aussi galvanisant qu'il l'escomptera, il ne fera néanmoins qu'accroître l'ambiguïté du mythe, faisant appel à deux traditions différentes: la défense organisée d'un côté, l'énergie anarchique de l'autre. Comme Pierre Cambon nous le rappelle, «il renvoie, d'un côté, à la résistance civile, spontanée, anonyme, souvent individuelle, de l'autre aux opérations effectuées par les corps francs, corps de volontaires régulièrement organisés, reconnus par le Ministère de la Guerre; soit, d'une part, à des actes parfaitement inorganisés, sporadiques, d'autre part, à des actions régulières et paramilitaires ${ }^{14}$.

7 La déclaration républicaine classique sera sans doute celle de Jean Jaurès dans son analyse de l'armée de la Troisième République, après la défaite de la guerre francoprussienne. Pour lui, il est impératif que la France redécouvre la vigueur et la force morale qui ont caractérisé, selon son imaginaire historique, les armées de la Révolution. Il fait comme Robespierre l'éloge du soldat ordinaire mais il voit dans les réformes de Carnot l'expression du génie révolutionnaire : l'amalgame, unissant des soldats aux qualités différentes dans une force unique, répond à la fois aux intérêts des politiques et de la communauté nationale dans son ensemble. La vision de la démocratie révolutionnaire au sein des bataillons - la discipline librement consentie par les soldats, l'élection des officiers - l'inspire. Par-dessus tout, il salue l'égalitarisme de la levée en masse qui, en temps de guerre, fait que chacun doit se rendre disponible sans distinction de son rang dans la société, que chacun a une tâche à accomplir pour sauver «la patrie en danger ». Bien que l'âge avancé, le sexe ou le mariage puissent exempter certains de monter au front, la Convention n'avait-elle pas déclaré que tous avaient un rôle à jouer? Presque cent ans après sa promulgation, les célèbres mots du décret continuent à résonner : « Dès ce moment jusqu'à celui où les ennemis auront été chassés du territoire de la République, tous les Français sont en réquisition permanente pour le service des armées. Les jeunes gens iront au combat; les hommes mariés forgeront les armes et transporteront les subsistances ; les femmes feront des tentes et serviront dans les hôpitaux; les enfants mettront le vieux linge en charpie; les vieillards se feront porter sur les places publiques pour exciter le courage des guerriers, prêcher la haine des rois et l'unité de la République " ${ }^{15}$. Telle est la vision qui fait vibrer le camp républicain, celle d'un peuple en armes répondant à l'appel de la nation. Comme l'écrit Jaurès, en exécutant les réformes de Carnot et de Dubois-Crancé, la Révolution a réussi à forger une armée proche du peuple, prête à se battre en son nom. Malgré le mépris du général prussien Von Moltke pour ces Français qui, en 1870, évoquent l'esprit de leurs prédécesseurs des années 1790, Jaurès croit encore en cet 
idéal qui fait de tous les hommes des soldats, sans nul conflit entre leurs responsabilités civiles et leurs devoirs militaires. Si la Révolution n'avait pas été interrompue, il soutient que la France du XIX siècle ne serait pas tombée sous le joug d'une armée professionnelle, que l'entraînement et l'éducation militaires auraient été centraux dans l'instruction et l'apprentissage des jeunes générations ${ }^{16}$.

Bien entendu, cette image du soldat républicain relève de la fiction, non seulement parce que de nombreux soldats de troupe sont requis plutôt que volontaires, mais aussi, de manière plus cruciale, parce que les qualités qu'ils sont supposés incarner sont celles d'une figure composite dessinée tout au long de la décennie révolutionnaire. L'enthousiasme et l'énergie caractérisent en grande partie les gardes nationaux et les premiers volontaires de 1791, des hommes qui, dans la plupart des cas, sont prêts, par amour de la Révolution et "de la chose publique ", à partir pour défendre les frontières. D'autre part, le savoir-faire et le dévouement, qualités qui distinguent les guerriers expérimentés, sont davantage le fait de beaucoup d'hommes qui, sous le Directoire, portent le message de la Révolution au-delà du Rhin ou qui accompagnent Bonaparte lors de la campagne d'Italie. Il y a parmi eux des individus dont la vie entière est marquée par l'engagement militant, des révolutionnaires dévoués qui s'engagent parce qu'ils veulent détruire les ennemis de la France et les forces de la réaction, des hommes qui prennent fait et cause pour l'armée. Auguste, par exemple, qui sert dans l'Armée des Vosges, écrit en ces termes à la société populaire dépendant de la section du faubourg Saint-Antoine (Paris) : «La clique sacerdotale et nobiliaire, ces loups voraces toujours nos ennemis, auraient-ils pu nous troubler en interrompant notre constitution que nous devrions avoir il y a longtemps? $"{ }^{17}$. Mais d'autres témoignages suggèrent que ces militants représentent une minorité de la troupe dont la majorité est plus intéressée par la nourriture, la boisson et les besoins du quotidien, si difficiles à assouvir au sein des régiments. En outre, le soldat mythifié se distingue par sa citoyenneté et par le fait que lui et ses pairs sont égaux face au sergent-recruteur, tous étant également susceptibles d'être appelés au service de l'État. Cette égalité est assez réelle, mais elle devient plus évidente en 1793 puis avec la conscription instaurée par la loi Jourdan; elle n'a rien à voir avec l'état d'esprit des premiers volontaires. D'un autre côté, l'armée des années 1790 change rapidement de taille et de nature. Les volontaires, qui ont combattu et vaincu à Valmy les troupes professionnelles et aguerries de l'empereur d'Autriche, ont peu de choses en commun avec les demibrigades de la levée en masse, encore moins avec les armées victorieuses du Directoire. L'armée est plus motivée par la fierté de la profession et la camaraderie militaire que ne l'ont été les premières unités de volontaires, inexpérimentées en $1792{ }^{18}$. Mais le mythe les a confondus en associant l'enthousiasme de jeunesse des gardes nationaux et l'égalité devant le sergent-recruteur à la fierté nationale de vaincre les armées de la coalition et à l'héroïsme de la garde napoléonienne. Aux yeux de ses nombreux admirateurs, l'armée des années 1790 est française avant d'être révolutionnaire, un symbole du triomphe national plutôt qu'un vecteur des valeurs républicaines. De plus, dans les années qui se sont écoulées de 1799 à 1870, l’armée française entreprend une série de réformes et de transformations. Elle en ressort bien différente de celle dont on se souvient avec une pointe de nostalgie. Sous Napoléon, les bataillons français sont devenus bien plus professionnels. Ils sont composés de conscrits provenant de toute l'Europe continentale ainsi que de Français qui ont encore en mémoire le sens de la loyauté vis-à-vis de la nation et du peuple. Les soldats se battent pour le gain et pour l'honneur plutôt que par idéalisme républicain. Ils se considèrent de plus en plus 
comme des militaires de carrière, condamnés à passer de longues années au sein des armées. Ils sont sensibles au charme des promotions et des honneurs et de plus en plus loyaux à leurs régiments et à leurs compagnons d'armes davantage qu'ils ne servent une idéologie ou la cause du peuple français ${ }^{19}$. Ils sont particulièrement bien entraînés aux tactiques et formations de combat. Leur savoir-faire inspire le respect, mais aussi la crainte des états-majors à travers l'Europe - d'où le désir chez certains, comme Stein en Prusse en 1806, d'imiter les Français en réformant leurs propres pratiques de recrutement, mais en leur conservant un caractère incontestablement allemand ${ }^{20}$. Mais s'ils sont de bons soldats, c'est grâce à leur professionnalisme, à leur entraînement, au commandement d'un grand chef militaire qui les conduit, les stimule et les inspire. Nul besoin désormais de leur suggérer que leurs qualités dépendent de leurs idéaux, de leur enthousiasme spontané ou encore de leur égalité civile. De fait, ils ne peuvent plus aspirer à cette dernière puisque, sauf pour la conscription de 1799 qui inaugure la loi Jourdan, il n'y a pas une seule année sous le Consulat ou sous l'Empire où l'on ne donne pas aux riches la possibilité d'acheter des remplaçants pour l'effectuer à leur place une politique que l'État justifie au nom de l'utilité publique. Ainsi, à partir de ventôse an VIII, la loi autorise le remplacement des conscrits des cinq classes pour des raisons d'incapacités physiques, de convenances personnelles ou d'intérêt public. Sont concernés " ceux qui ne pourraient supporter les fatigues de la guerre », les receveurs des postes ou les fabricants de munitions, ou encore les élèves de Polytechnique ou de l'École des Mines «qui seront plus utiles à l'État en continuant leur travail ou leurs études " ${ }^{21}$. La mémoire glorieuse évoquée au XIX ${ }^{\mathrm{e}}$ siècle et fondée sur l'aura de la victoire, l'image d'une armée qui a imposé la domination française de l'Atlantique à l'Oural, entretenue par ces vieux soldats qui retournent dans leurs foyers après Waterloo.

9 Une fois la paix rétablie, le pays, épuisé par les écrasantes levées des dernières années de l'Empire, est moins enclin à la conscription. Ainsi, la notion de soldat-citoyen est progressivement renvoyée à l'histoire puisqu'une série de réformes introduite par Gouvion Saint-Cyr en 1818, puis par Soult en 1832, rétablit la primauté d'une armée de métier dans laquelle la conscription joue un rôle secondaire. Pour les monarchistes et les bonapartistes, ce choix pose peu de problèmes car l'idéal de soldat-citoyen ne les a jamais réellement séduits. Plus intéressante sera la réaction des républicains du XIX siècle pour lesquels l'égalité sera un mantra et la mémoire de la Grande Révolution une partie de leur héritage. Leur ferme conviction des mérites d'une armée citoyenne ne peut être mise en doute. C'est un thème largement abordé dans la presse républicaine, dans Le National ou La Réforme, qui contribue dans une large mesure à la perpétuation du mythe du soldat-citoyen. «La force d'une armée dépend de l'intelligence et de l'instruction des individus qu'elle reçoit dans ses rangs. Une des causes principales de la supériorité des armées de la République et de l'Empire sur les armées de la coalition fut la réquisition qui, aidée de la levée en masse, fit passer sous les drapeaux, sans distinction de rang ni de fortune, la partie la plus vive, la plus ardente, la plus éclairée de la population française ». Tout en admettant qu'un manque d'expérience a entraîné les premiers échecs, l'article montre ensuite que ce fut cela même qui, en quelques années, a conduit à la gloire des campagnes napoléoniennes puisque, à travers «ses efforts héroïques", la réquisition a fourni à la France "les meilleurs soldats et les premiers généraux du monde $"{ }^{22}$. Mais cet enthousiasme pour la conscription ou pour une nouvelle "levée en masse» reste du domaine de la rhétorique. Par deux fois, les républicains sont au pouvoir dans la première moitié du XIX ${ }^{e}$ siècle, en 1830 puis en 
1848, et cependant ils ne font rien. Ils n'ont aucune envie de passer une loi en faveur d'une nouvelle conscription qui deviendra une question électorale le jour où la nation sera une nouvelle fois confrontée à une guerre de défense nationale en 1870. Et même alors, face à la perspective d'une défaite écrasante et humiliante, les conservateurs réussiront à s'opposer à l'abolition du remplacement et au projet que tout citoyen, quels que soient son statut social et ses revenus, paye sa quote-part de "l'impôt de sang $)(23$.

10 Cette mémoire, entretenue après 1845 , est aussi hybride par d'autres aspects. Elle est, d'une part, un mélange d'images républicaines contrastées - élans révolutionnaires ordonnés et spontanés, nation en armes -, mais, d'autre part, elle fait peu de différence entre les armées de la République et les hommes qui ont servi l'Empire. La fierté nationaliste se confond avec l'ardeur révolutionnaire, les uniformes de la garde nationale avec ceux de la garde impériale parce que la France d'après-guerre se bat pour mettre fin à la confusion et la diversité de l'héritage de plus de vingt ans de conflit. Ce legs doit être considéré dans tous ces aspects. Il est incarné par les vétérans qui, après la défaite, retournent en masse dans leurs villages où ils deviennent des figures emblématiques. Peu importe le régime qu'ils ont servi. Car si beaucoup restent fidèles à l'empereur, d'autres, qui ont servi sous les drapeaux depuis la conscription de 1799 , ou, qui se sont portés volontaires en 1791, ont d'abord consacré leur vie au service de la France. Contrairement aux décennies qui suivront les guerres du $\mathrm{XX}^{\mathrm{e}}$ siècle durant lesquelles les vétérans de l'armée deviendront des groupes de pression bien organisés réclamant l'attention et la gratitude de leurs concitoyens, leurs prédécesseurs ont une organisation des plus réduite. Mais ils sont des centaines de milliers, ce qui en soi leur donne de l'importance. Et lorsqu'en 1856, plus de quarante ans plus tard, on demandera aux communes de récompenser les vétérans des guerres napoléoniennes en leur offrant la médaille de Sainte-Hélène, 95 \% d'entre elles dans la Meurthe proposeront des noms ${ }^{24}$. On les admire pour leurs exploits et pour la notabilité acquise dans la vie de leur village. Beaucoup d'entre eux ont été élus maires dans leur commune de naissance, surtout sous Louis-Philippe. La plupart, figures emblématiques, cherchent la compagnie d'autres vétérans pour se remémorer leurs triomphes et leurs souffrances. Ils jouent un rôle primordial lors des veillées et des rassemblements villageois. On écoute leurs récits, leurs voyages avec avidité ; ces faits leur garantissent un statut certain au sein de la société rurale ${ }^{25}$.

11 Ils contribuent également à la vie politique sous la Restauration en influençant l'opinion publique. Une fois de plus, leur apport ne distingue pas entre la cause de la République et celle de l'Empire. Ils partagent la même animosité à l'encontre de la monarchie, de la Restauration, née de leurs années dans les régiments et confortée par les tentatives du nouveau régime de réintégrer dans l'armée des émigrés dignes de confiance et des royalistes tandis que sont mis à la retraite des officiers napoléoniens. L'armée est suspecte après les Cent Jours. Dans un premier élan, le nouveau régime met à la retraite le plus de soldats possible et efface les traditions militaires héritées de l'Empire. Le nombre d'officiers est réduit de 14000 , celui des hommes de rang fixé à 324 $000{ }^{26}$. Cela ne contribue pas à faire aimer la monarchie aux vétérans. Les anciens soldats jouent un rôle majeur dans le mouvement " néo-bonapartiste » qui émerge au début de la Seconde Restauration. À travers toute la France, on signale des slogans et des cris séditieux (« Vive l'empereur!» ou « À bas les fleurs de lys!»). Les émeutes et les manifestations ont presque toujours à leur tête des vétérans, et la convocation des conscrits devant le conseil de guerre, après la loi Gouvion-Saint-Cyr, est souvent 
accompagnée de chansons bonapartistes interprétées par de jeunes soldats ${ }^{27}$. Le but est de rassembler républicains et bonapartistes dans un mouvement commun d'opposition au sein duquel de vieux soldats se rappellent la gloire militaire pour laquelle ils se sont battus et minimisent leurs divergences politiques pour former une alliance pragmatique et évincer les Bourbons. Ils se considèrent comme des alliés naturels, unis par leur expérience militaire contre ceux qui défendent les privilèges et les vieilles élites. À Paris, lors de la Révolution de 1830, ils combattent côte à côte sur les barricades; en effet, les principaux leaders révolutionnaires sont des hommes qui se sont déjà battus pour la République et l'Empire, des vétérans des campagnes menées au nom du peuple français. Un docteur, qui soigne des blessés à Paris, constate que les deux tiers de ses patients ont participé aux guerres napoléoniennes ${ }^{28}$. Pour ceux-ci et pour la plupart de leurs camarades, le républicanisme et le bonapartisme sont devenus des versions alternatives d'un rêve populiste. Lors des combats contre les troupes de Charles X, les «Vive l'empereur!» se mêlent aux revendications en faveur du rétablissement de la République. Les anciens soldats se préoccupent moins d'obtenir une constitution que de prendre leur revanche sur la défaite de la France et de l'empereur en $1815^{29}$. La cause de la Révolution et celle de l'Empire se confondent avec celle de la nation.

Pour de nombreux jeunes Français qui ont combattu lors des dernières campagnes napoléoniennes, ou qui, dans les années qui suivent la Restauration, rêvent de gloire militaire, Napoléon incarne les nombreuses valeurs qui ont émergé du combat révolutionnaire: une société dans laquelle le statut social dépend du mérite, de la fonction et non de la naissance, dans laquelle tous les hommes sont égaux face à la loi et peuvent tous prétendre au bâton de maréchal, qu'ils soient nobles, riches bourgeois ou fils d'aubergiste comme l'était Murat ${ }^{30}$. Napoléon, bien entendu, contribue de manière flagrante à la naissance de sa propre légende et il aime insister sur l'égalité civique, surtout dans le domaine militaire. Car si son armée n'est plus celle de l'an II, elle possède encore en grande partie l'énergie de l'armée révolutionnaire, et si la gloire est partagée par tous, officiers et hommes de troupe, il en est de même de la souffrance. Dans ses discours et grâce aux arts qu'il protège, Napoléon assure son inscription dans la postérité, forgeant une imagerie qui le représente partageant avec ses hommes les tourments de la guerre et le succès des victoires. Ainsi, dans ses bulletins, produit de la guerre moderne, il loue les mérites de ses généraux et présente une armée courageuse et dévouée, tout en s'appropriant lui-même le rôle de pacificateur, de héros invincible. En 1797, on peut lire dans le premier numéro du Courrier de l'Armée d'Italie : «Bonaparte vole comme l'éclair et frappe comme la foudre [...] Il sait qu'il est des hommes dont le pouvoir n'a d'autres bornes que leur volonté quand la vertu des plus sublimes vertus seconde un vaste génie " ${ }^{31}$. De même, il se sert de la presse militaire lorsqu'il lui semble opportun de rappeler à l'opinion son identité révolutionnaire, son républicanisme et sa haine de la monarchie, qualités qui sont mises en avant à la veille de brumaire et servent après 1815 pour étayer un autre aspect de la légende napoléonienne : la perception de Bonaparte comme le digne successeur de la Révolution, comme le stabilisateur et l'héritier des idéaux et des principes de la République. Dans cette représentation de l'histoire, l'armée et la nation ne font qu'un, chacune d'elles renforçant la gloire de Napoléon. On organise des festivités pour vanter les armées, célébrer leurs victoires ou marquer les signatures des traités de paix. La musique militaire est systématiquement convoquée, revues et défilés militaires sont 
particulièrement valorisés, et l'on diffuse le message de l'inextricable union entre l'État et l'armée pour la défense de la nation et du peuple français ${ }^{32}$.

13 La peinture est le media favori de Napoléon pour son œuvre de propagande qui compte indubitablement parmi ses plus grandes réussites. Il s'intéresse personnellement aux œuvres d'art, qu'il encourage activement en organisant régulièrement des concours à l'occasion des Salons parisiens. Il préfère particulièrement les tableaux prenant pour thème l'histoire contemporaine, surtout les scènes de bataille, aux allégories et aux sujets classiques des années 1790. Aidé de son directeur général des musées, Dominique Vivant-Denon, il cherche à remédier aux erreurs de l'époque révolutionnaire. Denon a une grande influence; à chaque exposition, il oriente le choix de Napoléon dans les domaines suivants : acquisition des tableaux, sommes à payer, importance et valeur des prix et des honneurs attribués aux artistes ${ }^{33}$. Les célèbres tableaux de Gros et David, de Vernet et Géricault exaltent des images voluptueuses de gloire militaire en Égypte, dans les Alpes ainsi qu'à Iena et Marengo. Soutenus par les pensions du gouvernement, les artistes utilisent leurs talents pour représenter les succès glorieux et le rôle de Bonaparte sur le champ de bataille. Dans la première période de l'Empire, marquée par une succession de victoires et de conquêtes militaires soutenues par l'enthousiasme public, le fameux tableau de Vernet reproduisant la bataille de Marengo est très représentatif du genre. Selon Christopher Prendergast, «la description des combats obéit aux règles cardinales de la peinture historique : assujettir la violence de la guerre au pouvoir maîtrisé d'une composition esthétiquement ordonnée qui garantit pardessus tout précision, clarté et calme au milieu du chaos " ${ }^{34}$. Cette quiétude permet au peintre de s'appesantir sur le rôle du héros, d'insister sur la gratitude d'une armée en admiration devant la domination tactique de son général.

Il va sans dire que toutes les batailles ne se traduisent pas par une victoire pour la France et, avec le temps, les Français se laissent moins séduire par les images de propagande vantant des succès faciles et sans entraves. Le peuple se rend bien compte de l'agonie et de la souffrance des soldats. Les artistes officiels doivent maintenant faire face à un plus grand défi en présentant une image plus réaliste de la guerre sans amoindrir celle, sacrée, du héros, Napoléon sur le champ de bataille. Les deux chefsd'œuvre d'Antoine-Jean Gros, l'un consacré à Eylau en 1807, l'autre à Napoléon visitant les victimes de la peste à Jaffa en 1804, présentent la guerre et l'empereur sous un jour différent: un homme éprouvant de la compassion pour ses troupes malades, les accompagnant sur le champ de bataille au milieu des gisants. Eylau est en bien des points un désastre - de lourdes pertes humaines pour bien peu de profit - et Denon le reconnaît en choisissant ce thème pour une œuvre commémorative. Son but n'est pas de présenter une victoire glorieuse mais plutôt de s'appesantir sur la pathétique du champ de bataille au lendemain de la défaite, recouvert de cadavres déchiquetés et de corps mutilés. Denon explique « qu'il pense qu'il est préférable de choisir le lendemain du combat et le moment où l'empereur arrive sur le champ de bataille pour apporter son soutien et son réconfort à toutes les victimes sans distinction " ${ }^{35}$. Le thème abordé est autrement révélateur puisqu'il néglige les riches uniformes des officiers et insiste davantage sur le sort du soldat ordinaire. S'il est inspiré de l'héritage de la monarchie absolue qui a aidé à forger l'image de Louis XIV, il annonce aussi, comme dans l'œuvre de Géricault, un nouvel intérêt pour l'œuvre du commun des mortels ${ }^{36}$.

Sous Napoléon, ainsi que sous le Directoire, la conscription est si étendue, avec plus de deux millions de jeunes gens contraints à se présenter devant le sergent-recruteur, que 
l'armée fait partie de la vie quotidienne des familles françaises. L'enrôlement est un rite de passage à la fin de l'adolescence. En s'identifiant à ses hommes de troupe, l'empereur, de manière hautement symbolique, s'identifie au peuple français. Et, à la fin de son règne, grâce aux progrès technologiques de l'imprimerie, aux encres de couleurs vives désormais disponibles à bas prix, ce symbolisme pénètre chaque ville et chaque foyer partout dans le pays. Grâce au succès croissant des gravures sur bois, des tableaux colorés représentant des soldats, des petits tambours, des musiciens et des officiers de cavalerie décorent l'intérieur des paysans, des artisans et des ouvriers. Ils sont gravés sur des cartes achetées lors des fêtes rurales ou à des colporteurs de passage. Il en est de même pour les généraux, sans parler de Napoléon, qui deviennent dans les campagnes des figures familières posées sur le manteau des cheminées. L'empereur est représenté à cheval, vêtu de sa célèbre redingote, figure héroïque emblématique. Pour Jean-Claude Pellerin et les autres fabricants de ces images d'Épinal, la popularité des gravures militaires, des uniformes caractéristiques et des poses patriotiques, ouvre un nouveau marché lucratif. Auparavant, ils limitaient leur production à des images pieuses, des saints, des scènes du Nouveau Testament, autant d'icônes qui aboutissaient dans les foyers paysans. En produisant des millions d'images militaires pendant les guerres napoléoniennes, ils ajoutent à celles-ci des figures provenant de la tradition militaire telles que le sapeur, le tambour-major, la cantinière. Les pères et les mères reconnaissent leurs fils dans leurs splendides uniformes alors que les plus jeunes rêvent de rejoindre le combat de leurs aînés pour la défense de la nation. À la manière des grands tableaux des Salons parisiens, ces gravures bon marché jouent un rôle vital en rendant l'armée populaire et en abolissant le fossé qui sépare le soldat du civil ${ }^{37}$. Après 1815 , nombreuses sont ces productions jalousement gardées dans les fermes et les chaumières par les familles qui pleurent leurs fils disparus ou par les anciens soldats fiers de se rappeler les plus grands exploits de leur vie. Elles restent des biens précieux qui évoquent des souvenirs et servent d'objets de culte aux hommes scandalisés par les fêtes de la Restauration dévolues à la Passion du Christ et au Roi et par la publication d'images mièvres de Louis XVI et de Marie-Antoinette. Dans les jours sombres de la période 1815-1830, lorsque les républicains et les bonapartistes sont confrontés à la provocation et à la répression de l'État, ces gravures servent à raviver les souvenirs du passé et à alimenter la mémoire de l'armée de l'an II. En effet, dans de nombreuses régions françaises, elles participent d'un cérémonial construit autour de la conscription, une forme de culte privé, domestique, républicain, fervent et imprégné de folklore populaire ${ }^{38}$.

16 Après les journées de Juillet 1830, le climat politique change de manière spectaculaire. Louis-Philippe s'efforce de se concilier l'opinion publique et en appelle sans honte au mythe de la Révolution et de l'Empire. À l'instar de Napoléon Ier, le roi-citoyen rend populaire la légende des forces révolutionnaires. Par conséquent, les années 1830 deviennent une décennie propice à la diffusion de l'image des armées de la Révolution en direction des jeunes générations. Louis-Philippe a toutes les raisons de chercher à s'identifier à la Révolution : il s'est, après tout, rallié à l'appel de la "patrie en danger " et a servi comme chef des dragons à Valmy et à Jemmapes. Il s'appuie sur les valeurs patriotiques et la stabilité sociale pour construire la cause orléaniste à une époque où le nationalisme et l'identité nationale sont encore à peine à l'état embryonnaire dans la société française ${ }^{39}$. Valmy est transformé en un lieu de pèlerinage et ce qui a été comparativement une bataille mineure prend des proportions héroïques en devenant un tournant militaire de la décennie révolutionnaire. En 1831, le roi se rend à Valmy 
pour rencontrer les vieux soldats et les autochtones qui se rappellent le combat. Puis, en 1837, c'est au tour de la fiancée du futur duc d'Orléans de faire son pèlerinage sur le champ de la bataille ${ }^{40}$. En réponse, ses opposants républicains s'empressent de s'approprier Valmy, une bataille qui coïncide de manière fortuite avec la fondation de la République. C'est ainsi que cet événement a un impact majeur sur la mémoire des Français, symbolisant la nation en armes qui s'élève contre l'oppresseur - image que nourrit l'esprit romantique contemporain. Pour Michelet, «c'était le peuple à Valmy bien plus que l'armée [...] Sur toute cette jeune armée planait quelque chose comme une lueur héroïque, où le roi ne comprit rien [...]. Cette lueur était la foi ${ }^{41}$. Après 1879, les républicains radicaux en appellent à Valmy pour incarner l'esprit guerrier du peuple français, pétri de patriotisme et de zèle révolutionnaire. En effet, c'est une peinture de Valmy qui représente «la République » sur un des hauts reliefs du socle de la statue commandée par l'État ${ }^{42}$. Les batailles françaises du XX ${ }^{\mathrm{e}}$ siècle ravivent le mythe de Valmy auquel les républicains s'identifient. Ainsi, Jules Romains compare les lourdes pertes de la bataille de Verdun, en 1916, au sacrifice des volontaires de 1792. Romain Rolland, désespéré par l'occupation de son pays lors de la deuxième Guerre mondiale, s'écrie en 1946 : « Fils de la Révolution, êtes-vous encore capables d'entendre sans gêne les échos de la canonnade de Valmy? ${ }^{43}$

Louis-Philippe, en faisant appel au passé militaire de la France et au sentiment national, rêve d'unité et tente de gommer les divergences nées de l'histoire récente du pays. Sous la Restauration des Bourbons, toute allusion picturale ou théatrale aux exploits militaires et politiques de la Révolution et de l'Empire a été systématiquement censurée. Lorsque, en 1822, Louis-Philippe commande à Horace Vernet des tableaux représentant les batailles de Valmy et de Jemmapes, le jury du Salon les refuse. Cependant, après 1830, ces œuvres ont une seconde vie: les toiles de Vernet sont exposées par défi au Salon de 1831 - alors que zèle et passion révolutionnaires sont associés au Paris des Trois Glorieuses ${ }^{44}$. Le roi évoque ouvertement la mémoire de la Révolution pour souligner son propre héritage et convaincre la nation de sa légitimité. Pour le premier de ses grands projets artistiques, un nouveau décor pour l'hémicycle de la Chambre des députés, il choisit des thèmes révolutionnaires. Dans les années qui suivent, il ne cesse de rappeler à la France sa dette envers la Révolution. La décennie 1830 est marquée par l'abondance des tableaux exaltant la valeur des armées révolutionnaires et le sacrifice des troupes, le départ du volontaire étant un thème favori du topos romantique. En insistant sur le nationalisme de la Révolution, le nouveau régime cherche à la rendre respectable et à réintégrer son souvenir dans l'histoire nationale.

18 Mais si Louis-Philippe en appelle aux républicains et aux héritiers de la Révolution, il veut aussi obtenir l'appui de ceux qui entretiennent la mémoire napoléonienne s'attachant au Napoléon qui a offert aux Français de si nombreux et si mémorables triomphes militaires. Dans son projet de transformation du château de Versailles en musée national, il prévoit de créer une galerie des batailles, immense galerie de tableaux représentant des scènes historiques du Moyen-Âge à l'époque contemporaine afin de commémorer le génie militaire de la France à travers les époques. Le lieu n'est pas choisi au hasard: de tous les palais royaux, Versailles est celui qui représente la puissance par son gigantisme et sa somptuosité. On y redore le blason des rois, leurs gestes y sont mis en scène; pour reprendre les mots de Sylvia Brouillet, "c'est un miroir de la grandeur du prince [...], un espace privilégié de représentation du pouvoir $\gg{ }^{45}$. La galerie elle aussi traite du pouvoir, celui que l'on affiche sur le champ de 
bataille. Sept toiles sont consacrées à la période qui a débuté en 1789 , dont cinq aux seules guerres napoléoniennes. La bataille d'Austerlitz, moment phare de l'épopée, peinte par François Gérard, utilise l'espace pour valoriser le génie militaire de Napoléon, qui divise l'armée ennemie en son milieu pour mieux l'anéantir. L'œuvre fait explicitement les louanges de l'empereur: son armée est triomphante, la coalition vaincue ; lui-même, fin stratège et homme du peuple, est salué sur le champ de bataille par un soldat expirant tandis qu'il contemple l'étendue de sa victoire ${ }^{46}$. Dans sa quête d'unité nationale, Louis-Philippe subsume la mémoire des guerres révolutionnaires et napoléoniennes en une seule entité, une croisade nationale héroïque, menée par le peuple français au nom de la France. Pour légitimer sa monarchie auprès du peuple, il recourt à maintes reprises aux images militaires et accorde à l'armée un rôle privilégié lors des cérémonies d'État. La gloire de la nation importe au peuple et comme le montrera le somptueux cérémonial du Retour des Cendres en 1840, le régime se donne beaucoup de mal pour ceux qui se sont battus pour l'empereur et sont fiers de son souvenir. Une fois encore, la dimension militaire est cruciale à l'occasion de cet événement qui associe nationalisme et nostalgie. La cérémonie est minutieusement chorégraphiée jusque dans le moindre détail : le cercueil recouvert du drapeau des ennemis vaincus, la précision militaire de la procession descendant les Champs-Élysées, le rôle primordial réservé aux gardes nationaux et aux vétérans de l'Empire, Oudinot et Molitor, deux maréchaux de Napoléon, portant le drap mortuaire, un cheval vide de cavalier harnaché de la selle de Bonaparte à Marengo, la dignité de la dernière demeure de l'empereur aux Invalides, tout cela amplifie le symbolisme nationaliste et nourrit la prégnance $d u$ mythe ${ }^{47}$. Mais cela pose aussi problème au régime puisque toute résurgence d'un culte napoléonien pourrait représenter une menace à l'ordre public et provoquer une recrudescence du militarisme. Le roi le comprend et ne voit pas sans une certaine appréhension le rapatriement des cendres de Napoléon. Des républicains s'interrogent davantage en contestant la sagesse du geste qui risque de réveiller les espoirs bonapartistes. Lamartine, par exemple, est convaincu que trop peu de temps s'est écoulé pour apaiser les passions dangereuses des Français. Il met en garde contre une telle concentration d'intérêt général et d'enthousiasme qui peut aboutir à glorifier la guerre et à remplacer le culte de la liberté par celui de la force ${ }^{48}$.

19 Sous Napoléon III, le culte de l'empereur prendra bien entendu de plus grandes proportions et le militarisme se développera en conséquence rapidement. La Monarchie de Juillet est plus mesurée. Son but est d'associer républicains et bonapartistes pour soutenir le régime, de tirer parti des images de la gloire passée et de faire l'éloge de l'héritage militaire de la Révolution et de l'Empire. Le soldat de l'an II et le conscrit du Consulat et de l'Empire ne font qu'un, un personnage composite, courageux et plein de zèle, révolutionnaire et patriote, discipliné et prêt au sacrifice. Il partage les vertus des deux moments, que les nationalistes croient incarnées par tous les Français en temps de guerre. Cette image est popularisée grâce à des artistes qui se consacrent à l'armée comme Vernet et Denis Raffet; ils profitent des nouvelles techniques de gravure sur bois pour produire quantité d'illustrations pleines de vie pour les très populaires livres d'histoire militaire publiés dans les années 1830. Ces derniers contiennent aussi des récits de la vie de Napoléon écrits par Jacques de Norvins et Laurent de l'Ardèche qui contribuent à diffuser le culte impérial auprès de nouvelles générations de lecteurs ${ }^{49}$. La sculpture de François Rude pour l'Arc de Triomphe, commandée par la Monarchie de Juillet pour vanter les triomphes militaires de Napoléon, reste cependant l'expression la plus célèbre de ce culte: le panneau, 
achevé entre 1833 et 1836, communément appelé « La Marseillaise », dépeint ce qu'il y a de plus romantique dans l'imagerie révolutionnaire, c'est-à-dire le départ des volontaires de 1792. Il est accompagné de trois autres panneaux consacrés aux guerres napoléoniennes : la victoire de Wagram (1809), la résistance française (1814), la paix rétablie (1815). Cependant, de façon ironique sur le plus napoléonien des monuments, c'est l'œuvre de Rude qui résume le mieux la rébellion des Français et la mobilisation de la nation pour la guerre. Cette image, passionnément révolutionnaire, sera reproduite dans des centaines de livres scolaires sous la Troisième République et, pendant la première Guerre mondiale, apparaîtra sur les affiches appelant à la victoire ou à des souscriptions. La plus célèbre de ces affiches, pour la Banque Nationale de Crédit en 1916, illustre la continuité de la tradition militaire française : différentes générations de soldats de la République, de l'Empire ou de la Monarchie défilent en flots sous l'arc de Triomphe, appelés une fois encore par les hommes de la Première République, ceux qui ont répondu avec empressement à l'appel de la patrie en danger, à se rallier aux couleurs nationales ${ }^{50}$. Le soldat de l'an II fait non seulement partie de l'iconographie de l'armée française, mais aussi de la représentation de la nation elle-même.

\section{NOTES}

1.Cet article est une version développée d'un exposé sur « Les soldats de l'an II et le souvenir républicain ", présenté en août 2000 devant la Commission internationale d'histoire de la Révolution française lors du 19e Congrès des Sciences historiques à Oslo. Une partie de l'argumentaire a été développé dans une contribution au livre coordonné par Michel BIARD, Annie CRÉPIN et Bernard GAINOT, La plume et le sabre. Volume d'hommages offerts à Jean-Paul Bertaud, Paris, Publications de la Sorbonne, 2002, pp. 345-352. Je veux exprimer toute ma gratitude à la British Academy qui m'a accordé une Small Research Grant, aide qui a rendu possible les recherches nécessaires à cet article. 2.Christian AMALVI, "Nationalist responses to the Revolution », dans Robert TOMBS (dir.), Nationhood and Nationalism in France from Boulangism to the Great War, 1889-1918, Londres, 1991, p. 39.

3.Joseph SERVAN, Le soldat-citoyen, ou Vues patriotiques sur la manière la plus avantageuse de pourvoir à la défense du royaume, Paris, 1780, pp. 451-452.

4.Ibid., pp. 456-457.

5.Jean-Paul BERTAUD, La Révolution armée : les soldats-citoyens de la Révolution française, Paris, 1979, p. 93.

6.Albert soBoul, Les soldats de l'an II, Paris, 1959, pp. 24-27.

7.Thomas HIPPLER, «Service militaire et intégration nationale pendant la Révolution française ", Annales historiques de la Révolution française, $\mathrm{n}^{\circ} 329,2002$, pp. 3-6.

8.André CORVISIER, L'armée française de la fin du dix-septième siècle au ministère de Choiseul : le soldat, 2 volumes, Paris, 1964, vol. 1, pp. 179-180. Pour une étude plus détaillée des formes variées de recrutement habituellement utilisées au début du siècle, voir Georges GIRARD, Racolage et milice (1701-1715), Paris, 1921, pp. 75-161. 
9.Alan FORREST, Conscripts and Deserters. The Army and French Society during the Revolution and Empire, New-York, 1989, pp. 43-73. Pour le Sud-Ouest, région remarquable pour son haut niveau de résistance, les problèmes rencontrés par les autorités civiles après la mise en place de la conscription en l'an VI sont étudiés par Louis BERGÈs, Résister à la conscription (1798-1814). Le cas des départements aquitains, Paris, 2002.

10.John LYNN, The Bayonets of the Republic. Motivation and Tactics in the Army of Revolutionnary France (1791-1794), Urbana (Illinois), 1984, pp. 124-162.

11.Annie JOURDAN, « Robespierre and revolutionary heroism », dans Colin HAYDON et William DOYLE (dir.), Robespierre, Cambridge, 1999, p. 60.

12.Maximilien ROBESPIERRE, Cuvres complètes, Paris, rééd. 2001, volume 7, p. 263.

13.Appel de Gambetta, le 21 septembre 1870, dans André ROSSEL, 1870. La première guerre, par l'affiche et l'image, Paris, 1970.

14.Pierre CAMBON, Francs-tireurs et corps francs en 1870-1871: mythe et réalité, mémoire de maîtrise, Université Paris I - Sorbonne, 1976.

15.Décret du 23 août 1793.

16.Jean JAURÈs, L'armée nouvelle, édition Jean-Noël Jeanneney, Paris, 2 volumes, 1992, tome II, p. 248.

17.Louis Georges PÉLISSIER, Lettres de soldats (1792-1793), extrait de la Revue alsacienne, Paris, 1891, p. 5.

18. Albert MEYNIER, «L'armée en France sous la Révolution et le Premier Empire », Revue d'études militaires, 1932, pp. 17-20.

19.John LYNN, « Toward an army of honor : the moral evolution of the French army (1789-1815) », French Historical Studies, n 16, 1989, pp. 152-173.

20.John BREUILLY, « Napoleonic Germany and State-formation », dans Michael RowE (éd.), Collaboration and Resistance in Napoleonic Europe. State-formation in an Age of Upheaval, c. 1800-1815, Londres, 2003, p. 122.

21.Bernard SCHNAPPER, Le remplacement militaire en France. Quelques aspects politiques, économiques et sociaux du recrutement au XIXe siècle, Paris, 1968, p. 20.

22.Le National, 9 avril 1841, dans Annie CRÉPIN, La conscription en débat ou le triple apprentissage de la nation, de la citoyenneté, de la république (1798-1889), Arras, 1998, p. 92. 23.B. SCHNAPPER, op. cit., p. 271, note 21.

24.Françoise JOB, « Les anciens militaires de la République et de l'Empire dans le département de la Meurthe en 1857 et la médaille de Sainte-Hélène ", Le pays lorrain, $\mathrm{n}^{\circ}$ 61, 1981, p. 97.

25.David HOPKIN, Soldier and Peasant in French Popular Culture (1766-1870), Londres, 2003, pp. 282-284.

26.Pamela PILBEAM, The 1830 Revolution in France, Londres, 1991, p. 18.

27.Bernard MÉNAGER, Les Napoléon du peuple, Paris, 1988, pp. 19-25.

28.David PINKNEY, The French Revolution of 1830, Princeton (New Jersey), 1972, p. 271.

29.Jean-Lucas DUBRETON, Le culte de Napoléon (1815-1848), Paris, 1960, pp. 273-275.

30.Louis BERGERON, France under Napoleon, Princeton (New Jersey), 1981, p. 65.

31.Jean TUlARD, Le mythe de Napoléon, Paris, 1971, p. 31.

32.Voir, par exemple, l'ordre du cérémonial des différentes fêtes consulaires et impériales dans le Calvados, particulièrement la militarisation de la fête anniversaire de la Fondation de la République, organisée chaque année dans chaque commune du département pour célébrer le 14 Juillet (A.D. Calvados, M 2786). 
33.Yveline CANTAREL-BESSON, Claire CONSTANS et Bruno FOUCART (éd.), Napoléon, images et histoire : peintures du Château de Versailles (1789-1815), Paris, 2001, p. 99.

34.Christopher PRENDERGAST, Napoleon and History Painting: Antoine-Jean Gros's « La bataille d'Eylau ", Oxford, 1997, pp. 92-93.

35.David O'BRIEN, " Propaganda and the Republic of the Arts in Antoine-Jean Gros's Napoleon visiting the Battlefield of Eylau the Morning after the Battle ", French Historical Studies, $\mathrm{n}^{\circ} 26,2003$, p. 295.

36. Une excellente étude sur le processus de fabrication de l'image publique du roi et son adéquation aux attentes du public est proposée par Peter BURKE, The Fabrication of Louis XIV, New Haven (Connecticut), 1992.

37.R. PERROUT, Trésors des images d'Épinal, Barembach, 1985, p. 165.

38.Marie-Noëlle DENIS, « Les souvenirs de conscription en Alsace, témoins d'un culte civique domestique ", dans Maurice AGULHON (éd.), Cultures et folklores républicains, Paris, 1995, pp. 253-262.

39.Sudhir HAZAREESINGH, Political Traditions in Modern France, Oxford, 1994, p. 125.

40.Louis BERGÈs, Valmy, le mythe de la République, Toulouse, 2001, pp. 81-82.

41.Annie CRÉPIN, « Le mythe de Valmy », dans Michel vovelle (éd.), Révolution et République : l'exception française, Paris, 1974, p. 470.

42. Marie-Claude CHAUDONNERET, « Le mythe de la Révolution », dans Philippe BORDES et Michel RÉGIS (éd.), Aux armes et aux arts! Les arts de la Révolution (1789-1799), Paris, 1988, p. 317.

43.Emmanuel hublot, Valmy ou la défense de la nation par les armes, Paris, 1987, p. 403.

44. Michael MARRINAN, Painting Politics for Louis-Philippe. Art and Ideology in Orleanist France, New Haven (Connecticut), 1987, p. 78.

45.Sylvia BROUILLET, « De Versailles à Paris : Nicolas de Sainctot, chorégraphe de la monarchie en représentation (1711-1716) », dans Marie-France AUZÉPY et Joël CORNETTE (éd.), Palais et pouvoir de Constantinople à Versailles, Saint-Denis, 2003, p. 269.

46.M. MARRINAN, op. cit., p. 165.

47.Philip MANSEL, Paris between Empires (1814-1852), Londres, 2001, p. 369.

48. Michael Paul DRISKEL, As Befits a Legend. Building a Tomb for Napoleon (1840-1861), Kent (Ohio), 1993, p. 17.

49.Maurice SAMUELS, « Illustrated historiography and the image of the past in nineteenth-century France ", French Historical Studies, n² 26, 2003, pp. 258-269. 50.Daniel MORAN et Arthur WALDRON (éd.), The People in Arms. Military Myth and National Mobilization since the French Revolution, Cambridge, 2003, pp. 34-35.

\section{RÉSUMÉS}

Les armées de la Révolution française deviennent pour les générations à venir un point de référence cardinal, symbolisé par une gamme de qualités différentes et complémentaires - la conscription, bien entendu, l'égalité de tous devant le sergent-recruteur, le caractère personnel du service ; mais aussi des qualités humaines comme l'élan, la volonté, la générosité d'esprit et le 
patriotisme. Le soldat n'est pas soumis à la même critique que le politique, car il n'est pas perçu comme responsable de la même façon; et l'armée est évoquée et ses qualités louées par des régimes aussi divergents les uns des autres que la Monarchie de Juillet, le Second Empire et la Troisième République. Mais quelle est l'armée dont on se souvient? comment s'en souvient-on? et pourquoi le mythe de la levée en masse, de l'appel de la patrie en danger, s'avère-t-il si tenace ? Cet article retrace l'évolution de ce souvenir et examine les lectures que fera le dixneuvième siècle des soldats de la Révolution et de la nation en armes.

The Army of the Year II in modern memory: the levée-en-masse and the creation of a republican myth. The armies of the French Revolution would be a key point of reference for future generations, and would be characterised by a range of different and complementary qualities - by the fact of conscription, of course, the equality of all before the draft, and the requirement to serve in person; but also human qualities of flair, enthusiasm, generosity of spirit, and patriotism. The soldier is spared the criticism that is reserved for political figures; he does not share their responsibilities, and continues to enjoy a positive image under such different regimes as the July Monarchy, the Second Empire and the Third Republic. But which is the army that is being remembered? How is it remembered by future generations? And why does the myth of the levée en masse, of the response to the call of the patrie en danger, prove so resilient? This article traces the evolution of the army in public memory, examining the different depictions of the army and of the nation-in-arms that are encountered during the nineteenth century.

\section{INDEX}

Mots-clés : nation, patrie, an II, citoyen-soldat, commémoration, levée en masse, mythe napoléonien, volontaires 